\title{
Bioactive Pyrrole Alkaloids isolated from the Red Sea Marine Sponge Stylissa carteri
}

Ashraf N. E. Hamed ${ }^{1,2^{*}}$, Roland Schmitz ${ }^{3}$, Anja Bergermann ${ }^{4}$, Frank Totzke ${ }^{5}$, Michael Kubbutat $^{5}$, Werner E.G. Müller ${ }^{6}$, Diaa T. A. Youssef ${ }^{7}$, Mokhtar M. Bishr ${ }^{8}$, Mohamed S. Kamel ${ }^{2}$, RuAngelie Edrada-Ebel ${ }^{9}$, Wim Wätjen ${ }^{3,4}$, and Peter Proksch ${ }^{1}$

${ }^{1}$ Institut für Pharmazeutische Biologie und Biotechnologie, Heinrich-HeineUniversität, Universitätsstrasse 1, Geb. 26.23, 40225 Düsseldorf, Germany ${ }^{2}$ Department of Pharmacognosy, Faculty of Pharmacy, Minia University, 61519 Minia, Egypt

${ }^{3}$ Institut für Toxikologie, 1011007, Heinrich-Heine-Universität, Düsseldorf, Germany ${ }^{4}$ Martin-Luther-Universität Halle-Wittenberg, Faculty III, Institut für Agrar- und Ernährungswissenschaften, Weinbergweg 22, 06120 Halle/Saale, Germany ${ }^{5}$ ProQinase GmbH, Breisacher Str. 117, D-79106 Freiburg, Germany ${ }^{6}$ Institut für Physiologische Chemie, Universitätsmedizin der Johannes GutenbergUniversität Mainz, Düsbergweg 6, 55128 Mainz, Germany ${ }^{7}$ Department of Natural Products, Faculty of Pharmacy, King Abdulaziz University, Jeddah, 21589, Kingdom of Saudi Arabia ${ }^{8}$ Research and Development Department, Mepaco Company, Cairo, 11361, Egypt ${ }^{9}$ Strathclyde Institute of Pharmacy and Biomedical Science, Strathclyde University, The John Arbuthnott Building, 161 Cathedral Street, Glasgow G4 ONR, United Kingdom

*Corresponding author: Ashraf N. E. Hamed, Institut für Pharmazeutische Biologie und Biotechnologie, Heinrich-Heine-Universität, Universitätsstrasse 1, Geb. 26.23, 40225 Düsseldorf, Germany and Department of Pharmacognosy, Faculty of Pharmacy, Minia University, 61519 Minia, Egypt.

E-mail: ashrafnag@mu.edu.eg 


\section{Bioactive Pyrrole Alkaloids isolated from the Red Sea \\ Marine Sponge Stylissa carteri}

Abstract: Fifteen pyrrole alkaloids were isolated from the Red Sea marine sponge Stylissa carteri and investigated for their biological activities. Four of them were dibrominated $[(+)$ dibromophakelline, Z-3-bromohymenialdisine, $( \pm)$ ageliferin and 3,4-dibromo-1H-pyrrole-2-carbamide], nine compounds were monobrominated [(-) clathramide C, agelongine, (+) manzacidin A, (-) 3-bromomanzacidin D, Zspongiacidin D, Z-hymenialdisine, 2-debromostevensine, 2-bromoaldisine and 4bromo-1 $\mathrm{H}$-pyrrole-2-carbamide)] and finally, two compounds were non-brominated derivatives viz., [(E-debromohymenialdisine and aldisine $)]$. The structure elucidation of the isolated compounds were based on 1D and 2D NMR spectroscopic and MS studies, as well as by comparison with the literature. In-vitro, Z-spongiacidin D exhibited a moderate activity on (ARK5, CDK2-CycA, CDK4/CycD1, VEGFR-2, SAK and PDGFR) protein kinases. Furthermore, Z-hymenialdisine displayed a moderate effect on (ARK5, VEGFR-2, SAK and PDGFR) protein kinases. While, (-) clathramide $\mathrm{C}$ showed a moderate activity on AURORA-A. Moreover, Z-3bromohymenialdisine showed distinct inhibition of (AURORA-A, CDK4/CycD1, FAK, VEGFR-2, SAK and PDGFR) protein kinases. While, others showed only a marginal inhibitory activity e.g. agelongine and $(+)$ manzacidin $A$. The inhibition of these prominent protein kinases suggests a potential use of these compounds as cytostatic drugs. In L5178Y cell lines, the most effective secondary metabolites were (+) dibromophakelline and Z-3-bromohymenialdisine. Finally, Z-hymenialdisine, Z-3bromohymenialdisine and $( \pm)$ ageliferin exhibited the highest cytotoxic activity on HCT116 cell lines.

Keywords: Stylissa carteri; Sponge; pyrrole alkaloids; protein kinase; cytotoxicity.

\section{Introduction}

Marine sponges (Phylum: Porifera) have attracted substantial research interest because of their ecological importance and their production of a wide range of bioactive compounds for pharmacological use [1,2]. The Red Sea is a unique and largely unexplored marine ecosystem, where its sponges have been studied during 
the past two decades for their natural products and bioactive constituents, as well as for their ecological importance [3].

Stylissa carteri is one of the interesting sponges from the Red Sea. Several bromopyrrole alkaloids were isolated from it. A review demonstrated that these alkaloids showed promising biological activities, as hymenialdisine, first isolated in 1980 from the marine sponges of the genera Hymeniacidon, Acanthella, Axinella and Pseudaxinyssa. S. carteri is a well-known protein kinase inhibitor [4].

This sponge potently inhibited glycogen synthase kinase $3 \beta$, cyclin-dependent kinase 2 and cyclin-dependent kinase 5, whereas dibromocantharelline (another brominated sponge component) only displayed a significant inhibitory effect toward glycogen synthase kinase $3 \beta$ with $\mathrm{IC}_{50} 3 \mu \mathrm{mol}[5]$.

Moreover, 10-E-hymenialdisine and 10-Z-hymenialdisine showed a potent inhibition of RAF/MEK-1/MAPK cascade with $\mathrm{IC}_{50}$ values of 3 and $6 \mathrm{nM}$, respectively [6]. Both of these alkaloids also inhibited the growth of human LoVo tumor cells. Hymenialdisine competed with ATP for binding to distinct kinases, like cyclindependent kinases, glycogen synthase kinase-3 $\beta$ and casein kinase 1 [7]. Hymenialdisine inhibited interleukin-8 production in U937 cells by inhibition of nuclear factor-kappaB [8].

Furthermore, spongiacidin C (a pyrrole alkaloid was isolated from the marine sponge Stylissa massa) inhibited USP7, a deubiquitylating enzyme hydrolyzing the isopeptide bond at the C-terminus of ubiquitin. This potential cancer target was inhibited with $\mathrm{IC}_{50} 3.8 \mu \mathrm{M}[9]$.

Finally, the brominated alkaloids viz., debromohymenialdisine, hymenialdisine and 3-bromohymenialdisine of Axinella carteri exhibited cytotoxic activities on L5178Y mouse lymphoma cell lines. They displayed $E D_{50}$ values; $1.8,3.9$ and 3.9 $\mu \mathrm{g} / \mathrm{mL}$, respectively [10].

Therefore, this study aimed the investigation of the biological activities of the isolated alkaloids from $S$. carteri viz., in-vitro protein kinases activities and cytotoxic effects using two different cell lines.

\section{Material and methods}

\subsection{Animal material}


The sponge Stylissa carteri (syn. Axinella carteri) [Phylum: Porifera, Class: Demospongiae, Order: Halichondrida, Family: Dictyonellidae, Genus: Stylissa, Species: S. carteri] was collected in June 2006 at a depth of $12 \mathrm{~m}$, from the Red Sea, Hurghada, Egypt. It was identified by Prof. van Soest, RWM, (Zoological Museum; Amsterdam, The Netherlands) for the identification of the sponge. It is a reddish orange flabellate sponge. The sponge material was immersed in ethanol immediately after collection. A voucher specimen was kept in ethanol under registration number ZMAPOR 19838 at the Zoological Museum, Amsterdam, The Netherlands.

\subsection{Chromatography and spectroscopic analyses}

\subsubsection{Vacuum liquid chromatography (VLC)}

It was performed on silica gel 60 (0.040-0.063 mm; Merck, Darmstadt, Germany). $n$ Hexane, $\mathrm{CH}_{2} \mathrm{Cl}_{2}$ and $\mathrm{MeOH}$ were used as mobile phases. Column chromatography was carried out on silica gel 60, sephadex LH-20 and reversed phase LiChroprep RP-18 (25-40 $\mu \mathrm{m}$, Merck). For silica gel column chromatography, varying ratios of $\mathrm{CH}_{2} \mathrm{Cl}_{2} / \mathrm{MeOH}$ were used as mobile phases. For sephadex $\mathrm{LH}-20$ column chromatography, the mobile phase was $100 \% \mathrm{MeOH}$. For RP-18 column chromatography, two mobile phases were used; either acetonitrile or acetonitrile/ $/ \mathrm{H}_{2} \mathrm{O}$ (3:7). TLC analysis was carried out using aluminum sheet precoated with silica gel 60 F254 (Merck, Darmstadt, Germany). The compounds were detected by their UV absorbance at 254 and $366 \mathrm{~nm}$.

\subsubsection{Analytical HPLC analysis}

The samples were injected into a HPLC system equipped with a photodiode array detector (Dionex, Munich, Germany). The routine detection was at 235, 254, 280 and $340 \mathrm{~nm}$. The separation column (125 X $4 \mathrm{~mm}$ ID) was prefilled with Eurosphere 100$5 \mathrm{C}-18,5 \mu \mathrm{m}$ (Knauer, Berlin, Germany) and flow rate $1 \mathrm{~mL} / \mathrm{min}$. The separation was achieved by applying a linear gradient from $90 \% \mathrm{H}_{2} \mathrm{O}(\mathrm{pH} 2.0$ Nanopure water using ortho-phosphoric acid $85 \%$ p.a., Merck) to $100 \% \mathrm{MeOH}$ over $40 \mathrm{~min}$. 
The separations were done on a LaChrom-Merck Hitachi HPLC machine, pump L7100, UV detector L-7400. The separation column ( 300 X $8 \mathrm{~mm} \mathrm{ID)}$ was prefilled with Eurosphere 100-5 C-18, $5 \mu \mathrm{m}$ (Knauer, Berlin, Germany), flow rate $5 \mathrm{~mL} / \mathrm{min}$, UV detection at $280 \mathrm{~nm}$ ). The compounds were eluted with a solvent system of nanopure $\mathrm{H}_{2} \mathrm{O} / \mathrm{MeOH}$ (gradient elution starting with a concentration of $10 \% \mathrm{MeOH}$ and increasing the concentration in a linear manner within 25 min up to $60 \%$ ).

\subsubsection{Medium Pressure Liquid Chromatography (MPLC)}

The separations were done on Büchi MPLC; Gradient molder with mixing chamber B-687, fraction collector: B-684, Column size: (ID $460 \times 26 \mathrm{~mm}$ ), was prefilled with reversed phase LiChroprep RP-18 (25-40 $\mu \mathrm{m}$, Merck), flow rate $3 \mathrm{~mL} / \mathrm{min}$. The mobile phases were either $\mathrm{H}_{2} \mathrm{O} / \mathrm{MeOH}$ (7.5:2.5) or $\mathrm{H}_{2} \mathrm{O} / \mathrm{MeOH}$ gradient elution. Mass spectra (ESI-MS) were recorded on a Thermo Finnigan LCQ DECA mass spectrometer coupled to an Agilent 1100 HPLC system equipped with a photodiode array detector. HRFT-MS was recorded on a LTQ-FT-MS-Orbitrap (Thermo Finnigan, Bremen, Germany). Optical rotation was determined on a Perkin-Elmer$241 \mathrm{MC}$ polarimeter.

\subsubsection{Spectroscopic analyses}

1D and 2D NMR spectra were recorded at $300{ }^{\circ} \mathrm{K}$ on either a Bruker ARX-500 or AVANCE DMX-600 NMR spectrometer. Samples were dissolved in different deuterated solvents, whose choice was dependent on the solubility of each compound.

\subsection{Extraction and isolation}

The dried fine powder of Stylissa carteri $(600 \mathrm{~g})$ was extracted exhaustively with methanol (4x, $3 \mathrm{~L}$ each) and concentrated to yield $(90 \mathrm{~g})$ residue. The resulting extract was dissolved in the least amount of demineralized water and partitionated with EtOAc and $n-\mathrm{BuOH}$, respectively. The extraction and fractionation were demonstrated in (Scheme 1a). 
The $n-\mathrm{BuOH}$-soluble material of $S$. carteri was concentrated under vacuum to afford $18 \mathrm{~g}$. It was subjected to column chromatography on sephadex LH-20 using methanol as mobile phase. Four fractions were obtained (I to IV). Fraction (I, $3.05 \mathrm{~g}$ ) was further subjected to reversed phase column chromatography (RP-18, 25-40 $\mu \mathrm{m}$, Merck) using acetonitrile as a mobile phase to yield compounds 1 (6 mg). Furthermore, fraction (II, $3.58 \mathrm{~g}$ ) was subjected to MPLC on reversed phase (RP-18, 25-40 $\mu \mathrm{m}$, Merck) using $\mathrm{H}_{2} \mathrm{O} / \mathrm{MeOH}$ gradient elution to give three subfractions (Subfr. II-1 to II-3). Compound 2 (12 mg) was precipitated in pure form from subfraction (Subfr. II-1). On the other hand, subfraction (Subfr. II-2, $50 \mathrm{mg}$ ) was further submitted to column chromatography on reversed phase (RP-18, 25-40 $\mu \mathrm{m}$, Merck) using acetonitrile/ $\mathrm{H}_{2} \mathrm{O}$ (3:7) as a mobile phase to give compounds 3 (5 mg) and 4 (2 mg), respectively. In addition to, subfraction (Subfr. II-3, $30 \mathrm{mg}$ ) was further purified on semi-preparative HPLC to obtain compound $5(8 \mathrm{mg})$. Moreover, fraction (III, $4.9 \mathrm{~g}$ ) was further subjected to MPLC using reversed phase (RP-18, 25-40 $\mu \mathrm{m}$, Merck) and $\mathrm{H}_{2} \mathrm{O} / \mathrm{MeOH}$ (7.5:2.5) as an isocratic elution system to yield eight subfractions (from Subfr. III-1 to III-8). Subfraction (Subfr. III-3, $80 \mathrm{mg}$ ) was further purified using semi-preparative HPLC to afford three compounds 6 (3 mg), 7 (2 mg) in addition to $8(4 \mathrm{mg})$, respectively. On the other hand, subfraction (Subfr. III-6, 30 $\mathrm{mg}$ ) was submitted to further purification using semi-preparative HPLC to give compound 9 (5 mg). Furthermore, subfraction (Subfr. III-7, $30 \mathrm{mg}$ ) was subjected to semi-preparative HPLC to give compound $10(7 \mathrm{mg})$. Finally, compound 11 (10 mg) was precipitated in pure form from subfraction (Subfr. III-8). Chromatographic fractionations of $n$-butanol fraction of $S$. carteri sponge were illustrated in (Scheme 1b).

While, the EtOAc soluble material of $S$. carteri was concentrated under vacuum to yield $(12 \mathrm{~g})$ residue. It was subjected to VLC on silica gel, using gradient elution consisting of different portions of $n$-Hexane/ $\mathrm{CH}_{2} \mathrm{Cl}_{2}$ to $\mathrm{CH}_{2} \mathrm{Cl}_{2} / \mathrm{MeOH}$. Elution started with $100 \% n$-Hexane and the $\mathrm{CH}_{2} \mathrm{Cl}_{2}$ concentrations were increased gradually till $100 \% \mathrm{CH}_{2} \mathrm{Cl}_{2}$ and then the $\mathrm{MeOH}$ concentrations were increased gradually till $100 \%$ $\mathrm{MeOH}$. Ten fractions were obtained (from I to X). Compound $12(3 \mathrm{mg}$ ) was precipitated as a pure substance from fraction (VII). Additionally, fraction (IX, 500 $\mathrm{mg}$ ) was further subjected to silica gel column chromatography using $\mathrm{CH}_{2} \mathrm{Cl}_{2} / \mathrm{MeOH}$ in gradient elution manner to yield seven subfractions (from Subfr. IX-1 to IX-7). Subfraction (Subfr. IX-4, $110 \mathrm{mg}$ ) was further purified by reversed phase column chromatography (RP-18, 25-40 $\mu \mathrm{m}$, Merck) using acetonitrile as a mobile phase to 
afford three compounds; 13 (8 $\mathrm{mg}), 14(6 \mathrm{mg})$ and 15 (2 mg), respectively. Chromatographic fractionations of EtOAc fraction of $S$. carteri sponge were shown in (Scheme 1c).

\subsection{Protein kinase assay}

Assays for the measurement of protein kinase activity were performed in 96-well FlashPlates (Perkin Elmer/NEN, Boston, MA, USA) in a $50 \mathrm{~mL}$ reaction volume. The reaction cocktail contained $20 \mathrm{~mL}$ assay buffer, $5 \mathrm{~mL}$ ATP solution (in demineralized water), $5 \mathrm{~mL}$ test compound (in 10\% DMSO), $10 \mathrm{~mL}$ substrate and $10 \mathrm{~mL}$ purified recombinant protein kinase. The final concentration of ATP was $1 \mathrm{mM}$. The assay for all enzymes contained $60 \mathrm{mM}$ HEPES-NaOH, $\mathrm{pH} 7.5,3 \mathrm{mM} \mathrm{MgCl}_{2}, 3 \mathrm{mM} \mathrm{MnCl}$, 3 $\mathrm{mM}$ Na-orthovanadate, $1.2 \mathrm{mM}$ DTT, $50 \mathrm{mg} / \mathrm{mL}$ PEG20000 and $1 \mathrm{mM}\left[\gamma^{33} \mathrm{P}\right]$-ATP (approximately 5 * $10^{5} \mathrm{cpm} /$ well).

The following substrates were used: glycogen synthase kinase 3 (GSK3) (1427): AKT1 serine-threonine kinase, tetra(LRRWSLG): AURORA serine/threonine kinases $A$ and $B$, MEK kinase 1: B-RAF-VE kinase, Histone H1: Cyclin-dependent kinase (CDK2/CycA), Rb-CTF: Cyclin-dependent kinase 4 CDK4/CycD1, P53-CTM :anti-casein kinase 2 alpha (CK2alpha1), Poly(Glu,Tyr) 4:1: [epidermal growth factor receptor (EGFR), ephrin type B receptor 4 (EPHB4), ERBB2 receptor protein tyrosine kinase, focal adhesion kinase (FAK), insulin like growth factor 1 receptor (IGF1-R), SRC tyrosine kinase and vascular endothelial growth factor receptor (VEGF-R2)], Casein: Polo-like kinase PLK-1, poly(Ala, Glu, Lys, Tyr)6:2:4:1: [INS-R and MET] and Casein: platelet derived growth factor (PDGFR-beta). Autophosphorylation was measured for ARK5 serine/threonine kinase, COT kinase and SAK kinase.

The assay for all enzymes contained $60 \mathrm{mM} \mathrm{HEPES-NaOH} \mathrm{(pH} \mathrm{7.5),} 3 \mathrm{mM}$ $\mathrm{MgCl}_{2}, 3 \mathrm{mM} \mathrm{MnCl}$, $3 \mu \mathrm{M}$ Na-orthovanadate, $1.2 \mathrm{mM}$ DTT, $50 \mu \mathrm{g} / \mathrm{mL}$ PEG20000, 1 $\mu \mathrm{M}\left[\gamma\right.$-33P]-ATP. The reaction mixtures were incubated at $30{ }^{\circ} \mathrm{C}$ for $80 \mathrm{~min}$ and stopped with $50 \mu \mathrm{L} 2 \%(\mathrm{v} / \mathrm{v}) \mathrm{H}_{3} \mathrm{PO}_{4}$. The plates were aspirated and washed two times with $200 \mu \mathrm{L}$ of $0.9 \%(w / v) \mathrm{NaCl}$ or $200 \mu \mathrm{L} \mathrm{H}_{2} \mathrm{O}$. Incorporation of $\gamma^{33} \mathrm{P}$ was determined with a microplate scintillation counter (Microbeta Trilux, Wallac). All assays were performed with a Beckman Coulter/Sagian robotic system [11,12]. 


\subsection{Cytotoxicity test}

The cytotoxicity was determined by using two different cell lines:

\subsubsection{L5178Y cell lines}

They were grown in Eagle's minimal essential medium supplement with 10\% horse serum in roller tube culture. The medium contained $100 \mathrm{units} / \mathrm{mL}$ penicillin and 100 $\mu \mathrm{g} / \mathrm{mL}$ streptomycin. The cells were maintained in a humidified atmosphere at $37^{\circ} \mathrm{C}$ with $5 \% \mathrm{CO}_{2}$. An aliquot of $50 \mu \mathrm{L}$ cell suspension (3750 cells) was pipetted into each cavity of a 96-well microtiter plate together with $50 \mu \mathrm{L}$ of the compounds in EMEM ( 3 to $10 \mu \mathrm{g} / \mathrm{mL})$ and incubated for $72 \mathrm{~h}\left(37{ }^{\circ} \mathrm{C}, 5 \% \mathrm{CO}_{2}\right)$. A solution of $3-(4,5-$ dimethylthiazol-2-yl)-2,5-diphenyltetrazolium bromide (MTT) was prepared at 5 $\mathrm{mg} / \mathrm{mL}$ in phosphate buffered saline (PBS; $1.5 \mathrm{mM} \mathrm{KH}_{2} \mathrm{PO}_{4}, 6.5 \mathrm{mM} \mathrm{Na}_{2} \mathrm{HPO}_{4}, 137$ $\mathrm{mM} \mathrm{NaCl}, 2.7 \mathrm{mM} \mathrm{KCl} ; \mathrm{pH} 7.4$ ) and from this solution, $20 \mu \mathrm{L}$ was pipetted into each well. The yellow MTT penetrates the healthy living cells and in the presence of mitochondrial dehydrogenases, MTT is transformed to its blue formazan complex. After an incubation period of $3 \mathrm{~h}\left(37^{\circ} \mathrm{C}, 5 \% \mathrm{CO}_{2}\right)$, the medium was centrifuged (15 $\min , 20^{\circ} \mathrm{C}, 210 \mathrm{xg}$ ) with $200 \mu \mathrm{L}$ DMSO and the cells were lysed to liberate the formed formazan product. After thorough mixing, the absorbance was measured at $520 \mathrm{~nm}$ using a scanning microtiter-well spectrophotometer. The colour intensity is correlated with the number of viable cells $[13,14]$. All experiments were carried out in triplicates and repeated three times. As controls, media with $0.1 \%$ DMSO were included in the experiments.

\subsubsection{HCT116 cell lines}

They were evaluated according to Mosmann [14] with slight modifications [15].

\subsection{Statistical analyses}

All data are given as mean +/- SD. The significance of changes in the test responses was assessed using analysis of variance (GraphPad Prism: version 5.0, La Jolla, USA). Statistical significance was assessed by unpaired Student's $(t)$ test, differences were considered to be significant at $p<0.05$ and indicated as "*". 


\section{Results}

\subsection{Isolation of the pyrrole alkaloids}

From the Red Sea sponge S. carteri, fifteen compounds were isolated. All isolated compounds were identified by comparison with different techniques of spectroscopy (UV, MS and NMR) data with those in the literature: (-) clathramide C (1) [16], agelongine (2) [17], (+) manzacidin A (3) [18], (-) 3-bromomanzacidin D (syn. N-

methylmanzacidin C) (4) [19], (+) dibromophakelline (5) [20], $E-$ debromohymenialdisine (6) [21], Z-spongiacidin D (syn. axinohydantoin) (7) [22], Zhymenialdisine (8) [21], Z-3-bromohymenialdisine (syn. Spongiacidin A) (9) [21], 2debromostevensine (syn. 2-debromoodiline) (10) [21], ( \pm ) ageliferin (11) [23], 3,4dibromo-1H-pyrrole-2-carbamide (12) [24], aldisine (13) [25], 2-bromoaldisine (14) [25] and 4-bromo-1H-pyrrole-2-carbamide (15) [26-28]. The chemical structures are shown in (Figure 1).

\subsection{1 (-) Clathramide C (1) [16]}

Yellow residue.

UV (MeOH) $\lambda_{\max }: 221.5$ and $277.2 \mathrm{~nm}$.

$[\alpha]_{D}^{20}-7.0^{\circ}(c 0.11, \mathrm{MeOH})$.

${ }^{1} \mathrm{H}-\mathrm{NMR}\left(500 \mathrm{MHz}, \mathrm{CD}_{3} \mathrm{OD}\right): 7.03(1 \mathrm{H}, \mathrm{br} \mathrm{s}, \mathrm{H}-2), 6.84(1 \mathrm{H}, \mathrm{br} \mathrm{s}, \mathrm{H}-4), 4.04(1 \mathrm{H}, \mathrm{m}$, $\mathrm{H}-8), 2.28(2 \mathrm{H}, \mathrm{m}, \mathrm{H}-9), 4.57(1 \mathrm{H}, \mathrm{d}, \mathrm{J}=11.7 \mathrm{~Hz}, \mathrm{H}-11 \mathrm{a}), 4.35(1 \mathrm{H}, \mathrm{d}, J=11.7 \mathrm{~Hz}, \mathrm{H}-$ 11b), $7.99(1 \mathrm{H}$, br s, $\mathrm{H}-13)$ and $1.46(3 \mathrm{H}, \mathrm{s}, \mathrm{H}-15) .{ }^{13} \mathrm{C}-\mathrm{NMR}\left(125 \mathrm{MHz}, \mathrm{CD}_{3} \mathrm{OD}\right)$ : 160.4 (C, C-6), 154.0 (CH, C-13), 125.6 (CH, C-2), 123.4 (C, C-5), 118.3 (CH, C-4), 98.2 (C, C-3), $67.2\left(\mathrm{CH}_{2}, \mathrm{C}-11\right), 58.3(\mathrm{C}, \mathrm{C}-10), 52.4(\mathrm{CH}, \mathrm{C}-8), 32.1\left(\mathrm{CH}_{2}, \mathrm{C}-9\right)$, $24.2\left(\mathrm{CH}_{3}, \mathrm{C}-15\right)$ and $\mathrm{C}-16$ not detected. ESI-MS: $\mathrm{m} / \mathrm{z} 342$ and $344[\mathrm{M}, 1: 1]$ for $\mathrm{C}_{12} \mathrm{H}_{15}{ }^{79} \mathrm{BrN}_{4} \mathrm{O}_{3}$.

\subsubsection{Agelongine (2) [17]}

Yellow residue.

UV (MeOH) $\lambda_{\max }: 217.1,234.0$ and $271.8 \mathrm{~nm}$.

${ }^{1} \mathrm{H}-\mathrm{NMR}\left(500 \mathrm{MHz}, \mathrm{CD}_{3} \mathrm{OD}\right): 6.90(1 \mathrm{H}, \mathrm{br} \mathrm{s}, \mathrm{H}-2), 6.80(1 \mathrm{H}, \mathrm{br} \mathrm{s}, \mathrm{H}-4), 4.75(2 \mathrm{H}, \mathrm{t}$, $J=4.8 \mathrm{~Hz}, \mathrm{H}-8), 5.03(2 \mathrm{H}, \mathrm{t}, J=4.5 \mathrm{~Hz}, \mathrm{H}-9), 9.38(1 \mathrm{H}, \mathrm{s}, \mathrm{H}-11), 8.97(1 \mathrm{H}, \mathrm{d}, J=8.0 \mathrm{~Hz}$, $\mathrm{H}-13), 8.09(1 \mathrm{H}, \mathrm{t}, J=7.7 \mathrm{~Hz}, \mathrm{H}-14)$ and $9.01(1 \mathrm{H}, \mathrm{d}, J=6.0 \mathrm{~Hz}, \mathrm{H}-15) .{ }^{13} \mathrm{C}-\mathrm{NMR}(125$ $\mathrm{MHz}, \mathrm{CD}_{3} \mathrm{OD}$ ): 166.7 (C, C-16), 160.4 (C, C-6), 147.4 (CH, C-11), 147.0 (CH, C-13), 
146.5 (CH, C-15), 140.2 (C, C-12), 128.8 (CH, C-14), 125.6 (CH, C-2), 123.0 (C, C5), $118.5(\mathrm{CH}, \mathrm{C}-4), 98.2(\mathrm{C}, \mathrm{C}-3), 63.6\left(\mathrm{CH}_{2}, \mathrm{C}-8\right)$ and $61.8\left(\mathrm{CH}_{2}, \mathrm{C}-9\right)$. ESI-MS: $\mathrm{m} / \mathrm{z}$ 340 and $342[\mathrm{M}, 1: 1]$ for $\mathrm{C}_{13} \mathrm{H}_{13}{ }^{79} \mathrm{BrN}_{2} \mathrm{O}_{4}$.

\subsection{3 (+) Manzacidin A (3) [18]}

Yellow residue.

UV (MeOH) $\lambda_{\max }: 203.8$ and $275.8 \mathrm{~nm}$. $[\alpha]_{D}^{20}+11.6^{\circ}(c 0.67, \mathrm{MeOH})$.

${ }^{1} \mathrm{H}-\mathrm{NMR}\left(600 \mathrm{MHz}\right.$, DMSO- $\left.d_{6}\right): 12.56(1 \mathrm{H}, \mathrm{br} \mathrm{s},-\mathrm{NH}-1), 7.22(1 \mathrm{H}, \mathrm{br} \mathrm{s}, \mathrm{H}-2), 6.90$ (1H, br s, H-4), $4.27(1 \mathrm{H}, \mathrm{d}, \mathrm{J}=11.0 \mathrm{~Hz}, \mathrm{H}-8 \mathrm{a}), 4.21(1 \mathrm{H}, \mathrm{d}, \mathrm{J}=11.0 \mathrm{~Hz}, \mathrm{H}-8 \mathrm{~b}), 2.12$ $(1 \mathrm{H}, \mathrm{dd}, J=5.4,14.2 \mathrm{~Hz}, \mathrm{H}-10 \mathrm{eq}), 1.80(1 \mathrm{H}, \mathrm{dd}, J=10.7,13.6 \mathrm{~Hz}, \mathrm{H}-10 \mathrm{ax}), 4.36(1 \mathrm{H}$, d, J=11.0 Hz, H-11), $7.90(1 \mathrm{H}, \mathrm{s}, \mathrm{H}-13), 9.55(1 \mathrm{H}, \mathrm{br} \mathrm{s}, \mathrm{H}-14)$ and $1.28(3 \mathrm{H}, \mathrm{s}, \mathrm{H}-15)$. ${ }^{13} \mathrm{C}-\mathrm{NMR}\left(150 \mathrm{MHz}, \mathrm{CD}_{3} \mathrm{OD}\right.$ ): 174.2 (C, C-16), 160.4 (C, C-6), 151.3 (CH, C-13), $125.3(\mathrm{CH}, \mathrm{C}-2), 123.4$ (C, C-5), $118.3(\mathrm{CH}, \mathrm{C}-4), 98.1$ (C, C-3), $69.0\left(\mathrm{CH}_{2}, \mathrm{C}-8\right)$, 54.0 (C, C-9), $51.6(\mathrm{CH}, \mathrm{C}-11), 32.1\left(\mathrm{CH}_{2}, \mathrm{C}-10\right)$ and $24.2\left(\mathrm{CH}_{3}, \mathrm{C}-15\right)$. ESI-MS: $\mathrm{m} / \mathrm{z}$ 343 and $345[\mathrm{M}, 1: 1]$ for $\mathrm{C}_{12} \mathrm{H}_{14}{ }^{79} \mathrm{BrN}_{3} \mathrm{O}_{4}$.

\subsection{4 (-) 3-Bromomanzacidin D (N-Methylmanzacidin C) (4) [19]}

Yellow residue.

UV $(\mathrm{MeOH}) \lambda_{\max }: 218.1$ and $276.3 \mathrm{~nm}$.

$[\alpha]_{D}^{20}-3.4^{\circ}\left(c 0.3, \mathrm{CHCl}_{3}\right)$.

${ }^{1} \mathrm{H}-\mathrm{NMR}\left(600 \mathrm{MHz}, \mathrm{CD}_{3} \mathrm{OD}\right): 7.04(1 \mathrm{H}, \mathrm{d}, J=1.5 \mathrm{~Hz}, \mathrm{H}-2), 6.85(1 \mathrm{H}, \mathrm{d}, J=1.5 \mathrm{~Hz}, \mathrm{H}-$ 4), $4.58(1 \mathrm{H}, d, J=11.4 \mathrm{~Hz}, \mathrm{H}-8 \mathrm{a}), 4.30(1 \mathrm{H}, \mathrm{d}, J=12.3 \mathrm{~Hz}, \mathrm{H}-8 \mathrm{~b}), 2.63(1 \mathrm{H}, \mathrm{dd}, J=$ 5.7, $11.7 \mathrm{~Hz}, \mathrm{H}-10 \mathrm{eq}), 1.94$ (1H, dd, J= 11.4, $11.4 \mathrm{~Hz}, \mathrm{H}-10 \mathrm{ax}), 4.13$ (1H,dd, J= 5.0, $11.0 \mathrm{~Hz}, \mathrm{H}-11), 8.00$ (1H, br.s, $\mathrm{H}-13), 3.23(3 \mathrm{H}, \mathrm{s}, \mathrm{H}-15)$ and $1.47(3 \mathrm{H}, \mathrm{s}, \mathrm{H}-17) .{ }^{13} \mathrm{C}-$ NMR (150 MHz, CD ${ }_{3} \mathrm{OD}$ ): ${ }^{13} \mathrm{C}-\mathrm{NMR}\left(150 \mathrm{MHz}, \mathrm{CD}_{3} \mathrm{OD}\right.$ ): 174.3 (C, C-16), 160.7 (C, C-6), 154.0 ( $\mathrm{CH}, \mathrm{C}-13), 125.6$ (CH, C-2), 123.4 (C, C-5), 118.5 (CH, C-4), 98.3 (C, C-3), $67.1\left(\mathrm{CH}_{2}, \mathrm{C}-8\right), 58.3$ (C, C-9), $52.4(\mathrm{CH}, \mathrm{C}-11), 35.1\left(\mathrm{CH}_{2}, \mathrm{C}-10\right), 36.9\left(\mathrm{CH}_{3}\right.$, C-15) and $21.4\left(\mathrm{CH}_{3}, \mathrm{C}-17\right)$. ESI-MS: $m / z 357$ and 359 [M, 1:1] for $\mathrm{C}_{13} \mathrm{H}_{16}{ }^{79} \mathrm{BrN}_{3} \mathrm{O}_{4}$.

\subsection{5 (+) Dibromophakelline (5) [20]}

Brown residue.

UV $(\mathrm{MeOH}) \lambda_{\max }: 237.1$ and $289.4 \mathrm{~nm}$. $[\alpha]_{D}^{20}+3.0^{\circ}$ (c $\left.0.11, \mathrm{MeOH}\right)$. 
${ }^{1} \mathrm{H}-\mathrm{NMR}\left(500 \mathrm{MHz}, \mathrm{CD}_{3} \mathrm{OD}\right): 7.01(1 \mathrm{H}, \mathrm{s}, \mathrm{H}-3), 6.23(1 \mathrm{H}, \mathrm{s}, \mathrm{H}-6), 2.42(1 \mathrm{H}, \mathrm{m}, \mathrm{H}-$ 11a), $2.43(1 \mathrm{H}, \mathrm{m}, \mathrm{H}-11 \mathrm{~b}), 2.16(1 \mathrm{H}, \mathrm{m}, \mathrm{H}-12 \mathrm{a}), 2.19(1 \mathrm{H}, \mathrm{m}, \mathrm{H}-12 \mathrm{~b}), 3.63(1 \mathrm{H}, \mathrm{m}, \mathrm{H}-$ 13a) and $3.84(1 \mathrm{H}, \mathrm{m}, \mathrm{H}-13 \mathrm{~b}) .{ }^{13} \mathrm{C}-\mathrm{NMR}\left(125 \mathrm{MHz}\right.$, DMSO- $\left.d_{6}\right): 156.3$ (C, C-15), 153.7 (C, C-8), 125.0 (C, C-4), 114.8 (CH, C-3), 106.1 (C, C-5), 102.0 (C, C-2), 82.4 (C, C-10), $68.2(\mathrm{CH}, \mathrm{C}-6), 44.7\left(\mathrm{CH}_{2}, \mathrm{C}-13\right), 38.5\left(\mathrm{CH}_{2}, \mathrm{C}-11\right)$ and $19.0\left(\mathrm{CH}_{2}, \mathrm{C}-12\right)$. ESI-MS: $m / z$ 387, 389 and 391 [M, 1:2:1] for $\mathrm{C}_{11} \mathrm{H}_{11}{ }^{79} \mathrm{Br}_{2} \mathrm{~N}_{5} \mathrm{O}$.

\subsubsection{E-Debromohymenialdisine (6) [21]}

Yellow amorphous powder.

UV (MeOH) $\lambda_{\max }: 212.0,240.5$ and $362.1 \mathrm{~nm}$.

${ }^{1} \mathrm{H}-\mathrm{NMR}\left(500 \mathrm{MHz}\right.$, DMSO- $\left.d_{6}\right): 11.37(1 \mathrm{H}$, br s, -NH-1), $7.06(1 \mathrm{H}, \mathrm{br} \mathrm{s}, \mathrm{H}-2), 6.78$ $(1 \mathrm{H}, \mathrm{d}, \mathrm{J}=2.5 \mathrm{~Hz}, \mathrm{H}-3), 7.76(1 \mathrm{H}, \mathrm{br} \mathrm{s},-\mathrm{NH}-7), 3.19(2 \mathrm{H}, \mathrm{t}, \mathrm{J}=5.1 \mathrm{~Hz}, \mathrm{H}-8),(\mathrm{H}-9)$ under solvent peak from ${ }^{1} \mathrm{H}-{ }^{1} \mathrm{H}$ COSY, $9.52\left(2 \mathrm{H}, \mathrm{br} \mathrm{s},-\mathrm{NH}_{2}-14\right)$ and $10.40(1 \mathrm{H}, \mathrm{br} \mathrm{s}$, $\mathrm{NH}-15)$. ESI-MS: $m / z 245(\mathrm{M})$ for $\mathrm{C}_{11} \mathrm{H}_{11} \mathrm{~N}_{5} \mathrm{O}_{2}$.

\subsubsection{Z-Spongiacidin D (syn. Axinohydantoin) (7) [22]}

Yellow amorphous powder.

UV (MeOH) $\lambda_{\max }: 241.2,259.8$ and $360.1 \mathrm{~nm}$.

${ }^{1} \mathrm{H}-\mathrm{NMR}\left(500 \mathrm{MHz}, \mathrm{DMSO}-d_{6}\right): 12.16(1 \mathrm{H}, \mathrm{br} \mathrm{s},-\mathrm{NH}-1), 7.00(1 \mathrm{H}, \mathrm{s}, \mathrm{H}-3), 7.84(1 \mathrm{H}$, t, J=4.6 Hz, -NH-7), $3.19(2 \mathrm{H}$, br s, H-8), $3.19(2 \mathrm{H}, \mathrm{br} \mathrm{s}, \mathrm{H}-9), 9.58(1 \mathrm{H}, \mathrm{br} \mathrm{s},-\mathrm{NH}-13)$ and $10.55\left(1 \mathrm{H}\right.$, br s, -NH-15). ESI-MS: $m / z 324$ and $326[\mathrm{M}, 1: 1]$ for $\mathrm{C}_{11} \mathrm{H}_{9}{ }^{79} \mathrm{BrN}_{4} \mathrm{O}_{3}$.

\subsubsection{Z-Hymenialdisine (8) [21]}

Yellow amorphous powder.

UV (MeOH) $\lambda_{\max }: 210.1,262.1$ and $354.7 \mathrm{~nm}$.

${ }^{1} \mathrm{H}-\mathrm{NMR}\left(500 \mathrm{MHz}, \mathrm{DMSO}-d_{6}\right): 12.17(1 \mathrm{H}, \mathrm{br} \mathrm{s},-\mathrm{NH}-1), 6.77(1 \mathrm{H}, \mathrm{s}, \mathrm{H}-3), 7.93(1 \mathrm{H}, \mathrm{t}$, $\mathrm{J}=4.3 \mathrm{~Hz},-\mathrm{NH}-7), 3.17(2 \mathrm{H}$, br s, $\mathrm{H}-8), 3.17(2 \mathrm{H}$, br s, $\mathrm{H}-9), 9.10\left(2 \mathrm{H}, \mathrm{br} \mathrm{s},-\mathrm{NH}_{2}-14\right)$ and $10.56\left(1 \mathrm{H}\right.$, br s, -NH-15). ESI-MS: $m / z 323$ and $325[\mathrm{M}, 1: 1]$ for $\mathrm{C}_{11} \mathrm{H}_{10}{ }^{79} \mathrm{BrN}_{5} \mathrm{O}_{2}$.

\subsubsection{Z-3-Bromohymenialdisine (syn. Spongiacidin-A) (9) [21]}

Yellow amorphous powder.

UV (MeOH) $\lambda_{\max }: 201.8,271.6$ and $330.1 \mathrm{~nm}$.

${ }^{1} \mathrm{H}-\mathrm{NMR}\left(500 \mathrm{MHz}\right.$, DMSO- $\left.d_{6}\right): 13.06(1 \mathrm{H}$, br s, $-\mathrm{NH}-1), 7.87$ (1H, br s, $\left.-\mathrm{NH}-7\right), 3.18$ $\left(2 \mathrm{H}\right.$, br s, H-8), $3.18(2 \mathrm{H}, \mathrm{br} \mathrm{s}, \mathrm{H}-9), 8.81\left(2 \mathrm{H}, \mathrm{br} \mathrm{s},-\mathrm{NH}_{2}-14\right)$ and $10.38(1 \mathrm{H}, \mathrm{br} \mathrm{s}$, $\mathrm{NH}$-15). ESI-MS: $m / z$ 401, 403 and 405 [M, 1:2:1] for $\mathrm{C}_{11} \mathrm{H}_{9}{ }^{79} \mathrm{Br}_{2} \mathrm{~N}_{5} \mathrm{O}_{2}$. 


\subsubsection{2-Debromostevensine (syn. 2-Debromoodiline) (10) [21]}

Brown residue.

${ }^{1} \mathrm{H}-\mathrm{NMR}(500 \mathrm{MHz}$, DMSO-d 6 ): $11.91(1 \mathrm{H}$, br s, $-\mathrm{NH}-1), 7.00(1 \mathrm{H}, \mathrm{s}, \mathrm{H}-2), 8.50(1 \mathrm{H}$, t, J=5.7 Hz, -NH-7), $3.93(2 \mathrm{H}, \mathrm{t}, \mathrm{J}=5.1 \mathrm{~Hz}, \mathrm{H}-8), 6.16(1 \mathrm{H}, \mathrm{t}, \mathrm{J}=4.8 \mathrm{~Hz}, \mathrm{H}-9), 12.69$ $(1 \mathrm{H}, \mathrm{br} \mathrm{s},-\mathrm{NH}-12), 7.45\left(2 \mathrm{H}, \mathrm{br} \mathrm{s},-\mathrm{NH}_{2}-13\right), 11.91(1 \mathrm{H}, \mathrm{br} \mathrm{s},-\mathrm{NH}-14)$ and $6.87(1 \mathrm{H}$, s, $\mathrm{H}$-15). ESI-MS: $\mathrm{m} / \mathrm{z} 307$ and $309[\mathrm{M}, 1: 1]$ for $\mathrm{C}_{11} \mathrm{H}_{10}{ }^{79} \mathrm{BrN}_{5} \mathrm{O}$.

\subsubsection{1 ( \pm ) Ageliferin (11) [23]}

Brown residue.

$[\alpha]_{D}^{20} 0^{\circ}(c 0.11, \mathrm{MeOH})$

UV (MeOH) $\lambda_{\text {max }}: 228.7$ and $271.9 \mathrm{~nm}$.

${ }^{1} \mathrm{H}-\mathrm{NMR}\left(500 \mathrm{MHz}, \mathrm{CD}_{3} \mathrm{OD}\right): 6.96(1 \mathrm{H}, \mathrm{d}, J=1.5 \mathrm{~Hz}, \mathrm{H}-2), 6.96(1 \mathrm{H}, \mathrm{d}, J=1.5 \mathrm{~Hz}, \mathrm{H}-$ 2'), $6.85(1 \mathrm{H}, d, J=1.3 \mathrm{~Hz}, \mathrm{H}-4), 6.93(1 \mathrm{H}, \mathrm{d}, J=1.6 \mathrm{~Hz}, \mathrm{H}-4$ '), $3.50(1 \mathrm{H}, \mathrm{dd}, J=14.5$, $5.0 \mathrm{~Hz}, \mathrm{H}-8 \mathrm{a}), 3.77(1 \mathrm{H}, \mathrm{dd}, \mathrm{J}=14.5,4.4 \mathrm{~Hz}, \mathrm{H}-8 \mathrm{~b}), 3.33(1 \mathrm{H}, \mathrm{dd}, \mathrm{J}=13.6,4.5 \mathrm{~Hz}, \mathrm{H}-$ 8'a), 3.64 (1H, dd, J=13.9, 3.2 Hz, H-8'b), 2.17 (1H, m, H-9), 2.27 (1H, m, H-9'), 3.83 $(1 \mathrm{H}, \mathrm{d}, J=7.3 \mathrm{~Hz}, \mathrm{H}-10), 2.48(1 \mathrm{H}$, ddd, J=16.7, 7.9, $2.3 \mathrm{~Hz}, \mathrm{H}-10 \mathrm{\prime}$ ) $), 2.78$ (1H, ddd, $J=16.7,5.7,1.5 \mathrm{~Hz}, \mathrm{H}-10 \mathrm{\prime} b)$ and $6.79\left(1 \mathrm{H}\right.$, br s, H-15). ${ }^{13} \mathrm{C}-\mathrm{NMR}\left(125 \mathrm{MHz}, \mathrm{CD}_{3} \mathrm{OD}\right)$ : 163.2 (C, C-6), 162.9 (C, C-6'), 149.1 (C, C-13), 149.0 (C, C-13'), 127.6 (C, C-11), 127.3 (C, C-5), 127.2 (C, C-5'), 123.2 (CH, C-2), 123.1 (CH, C-2'), 122.9 (C, C-11'), 119.1 (C, C-15'), 114.3 (CH, C-4), 113.7 (CH, C-4'), 113.0 (CH, C-15), 97.7 (C, C-3), $97.5\left(\mathrm{C}, \mathrm{C}-3^{\prime}\right), 43.9(\mathrm{CH}, \mathrm{C}-9), 42.7\left(\mathrm{CH}_{2}, \mathrm{C}-8^{\prime}\right), 40.1\left(\mathrm{CH}_{2}, \mathrm{C}-8\right), 37.1\left(\mathrm{CH}, \mathrm{C}-9^{\prime}\right)$, $33.2(\mathrm{CH}, \mathrm{C}-10)$ and $\left.23.6\left(\mathrm{CH}_{2}, \mathrm{C}-10\right)^{\prime}\right)$. ESI-MS: $\mathrm{m} / \mathrm{z} 618,620$ and 622 [M, 1:2:1] for $\mathrm{C}_{22} \mathrm{H}_{24}{ }^{79} \mathrm{Br}_{2} \mathrm{~N}_{10} \mathrm{O}_{2}$.

\subsubsection{3,4-Dibromo-1H-pyrrole-2-carbamide (12) [24]}

White amorphous powder.

UV (MeOH) $\lambda_{\max }: 234.0$ and $276.2 \mathrm{~nm}$.

${ }^{1} \mathrm{H}-\mathrm{NMR}\left(500 \mathrm{MHz}\right.$, DMSO- $\left.d_{6}\right): 12.62(1 \mathrm{H}, \mathrm{br} \mathrm{s},-\mathrm{NH}-1), 6.90(1 \mathrm{H}, \mathrm{d}, \mathrm{J}=2.5 \mathrm{~Hz}, \mathrm{H}-5)$ and $7.58 \& 7.17\left(2 \mathrm{H}\right.$, br s, $\left.-\mathrm{NH}_{2}\right)$. ESI-MS: $m / z 266,268$ and 270 [M, 1:2:1] for $\mathrm{C}_{5} \mathrm{H}_{4}{ }^{79} \mathrm{Br}_{2} \mathrm{~N}_{2} \mathrm{O}$.

\subsubsection{Aldisine (13) [25]}

Yellow residue.

UV (MeOH) $\lambda_{\max }: 220.5,250.4$ and $303.9 \mathrm{~nm}$. 
${ }^{1} \mathrm{H}-\mathrm{NMR}\left(500 \mathrm{MHz}\right.$, DMSO- $\left.d_{6}\right): 12.13(1 \mathrm{H}$, br s, -NH-1), $6.97(1 \mathrm{H}, \mathrm{d}, \mathrm{J}=2.5 \mathrm{~Hz}, \mathrm{H}-2)$, $6.53(1 \mathrm{H}, \mathrm{d}, \mathrm{J}=2.5 \mathrm{~Hz}, \mathrm{H}-3), 2.69(2 \mathrm{H}, \mathrm{m}, \mathrm{H}-5), 3.34(2 \mathrm{H}, \mathrm{m}, \mathrm{H}-6)$ and $8.30(1 \mathrm{H}, \mathrm{br} \mathrm{s}$, -NH-7). ${ }^{13} \mathrm{C}-\mathrm{NMR}\left(125 \mathrm{MHz}, \mathrm{DMSO}-d_{6}\right): 194.3$ (C, C-4), 162.2 (C, C-8), 127.9 (C, C8a), 123.5 (C, C-3a), $122.3(\mathrm{CH}, \mathrm{C}-2), 109.5(\mathrm{CH}, \mathrm{C}-3), 43.5\left(\mathrm{CH}_{2}, \mathrm{C}-6\right)$ and 36.5 $\left(\mathrm{CH}_{2}, \mathrm{C}-5\right)$. El-MS: $m / z 164(\mathrm{M})$ for $\mathrm{C}_{8} \mathrm{H}_{8} \mathrm{~N}_{2} \mathrm{O}_{2}$.

\subsubsection{2-Bromoaldisine (14) [25]}

Yellow amorphous powder.

UV $(\mathrm{MeOH}) \lambda_{\max }: 233.9$ and $311.1 \mathrm{~nm}$.

${ }^{1} \mathrm{H}-\mathrm{NMR}\left(500 \mathrm{MHz}, \mathrm{DMSO}-d_{6}\right): 12.95(1 \mathrm{H}$, br s, -NH-1), $6.55(1 \mathrm{H}, \mathrm{s}, \mathrm{H}-3), 2.69(2 \mathrm{H}$, $\mathrm{m}, \mathrm{H}-5), 3.33(2 \mathrm{H}, \mathrm{m}, \mathrm{H}-6)$ and $8.37\left(1 \mathrm{H}\right.$, br s, -NH-7). ${ }^{13} \mathrm{C}-\mathrm{NMR}(125 \mathrm{MHz}$, DMSO$\left.d_{6}\right): 193.5$ (C, C-4), 161.3 (C, C-8), 129.4 (C, C-8a), 124.6 (C, C-3a), 111.2 (CH, C3), 105.2 (C, C-2), $43.4\left(\mathrm{CH}_{2}, \mathrm{C}-6\right)$ and $36.3\left(\mathrm{CH}_{2}, \mathrm{C}-5\right)$. El-MS: $m / z 242$ and 244 (M, 1:1) for $\mathrm{C}_{8} \mathrm{H}_{7}{ }^{79} \mathrm{BrN}_{2} \mathrm{O}_{2}$.

\subsubsection{4-Bromo-1H-pyrrole-2-carbamide (15) [26-28]}

Yellow residue.

UV $(\mathrm{MeOH}) \lambda_{\max }: 232.9$ and $269.9 \mathrm{~nm}$.

$\left.{ }^{1} \mathrm{H}-\mathrm{NMR}\left[500 \mathrm{MHz},\left(\mathrm{CD}_{3}\right)_{2} \mathrm{CO}\right)\right]: 10.90(1 \mathrm{H}$, br s, $-\mathrm{NH}-1), 6.84(1 \mathrm{H}, \mathrm{dd}, \mathrm{J}=2.8,1.5 \mathrm{~Hz}$, $\mathrm{H}-3), 7.01(1 \mathrm{H}$, dd, J=2.5, $1.5 \mathrm{~Hz}, \mathrm{H}-5)$ and 6.43 \& $7.14\left(2 \mathrm{H}\right.$, br s, $\left.-\mathrm{NH}_{2}\right) .{ }^{13} \mathrm{C}-\mathrm{NMR}$ (125 MHz, DMSO-d $\left.d_{6}\right): 161.1(\mathrm{C}=\mathrm{O}), 126.9$ (C, C-2), $121.2(\mathrm{CH}, \mathrm{C}-5), 112.0(\mathrm{CH}, \mathrm{C}-$ 3) and 94.8 (C, C-4). El-MS: $m / z 188$ and $190(\mathrm{M}, 1: 1)$ for $\mathrm{C}_{5} \mathrm{H}_{5}{ }^{79} \mathrm{BrN}_{2} \mathrm{O}$.

\subsection{Protein kinase inhibition}

Protein kinases are sensitive targets for various pharmacological purposes, e.g. VEGFR-2 is an important target in cancer therapy. This kinase was inhibited by distinct brominated pyrrole derivatives from S. carteri: Z-spongiacidin D (7), Zhymenialdisine (8), Z-3-bromohymenialdisine (9) as well as $E$ debromohymenialdisine (6) and 3,4-dibromo-1H-pyrrole-2-carbamide (12). Zspongiacidin $D(7)$ was shown to be the most active compound. It also inhibited AKT1, ARK5, AURORA-A, B-RAF-VE, CDK2/CycA, CDK4/CycD1, FAK, IGF1-R, SRC, VEGFR-2, COT, PLK-1, SAK and PDGFR-beta. A similar activity profile showed by Z-3-bromohymenialdisine (9), which inhibited AURORA-A, AURORA-B, CDK4/CycD1, FAK, SRC, VEGFR-2, COT, PLK1, SAK and PDGFR-beta. While, Z- 
hymenialdisine (8) was able to inhibit the activity of AKT1, ARK5, CDK2-CycA, CDK4/CycD1, FAK, VEGFR-2, COT, PLK1, SAK and PDGFR-beta. But, (-) clathramide $C(\mathbf{1})$, agelongine (2) $(+)$ manzacidin A (3), E-Debromohymenialdisine (6) and 3,4-dibromo-1 $\mathrm{H}$-pyrrole-2-carbamide (12) showed only a slight protein kinase inhibition. Finally, all other compounds were not active. The protein kinase inhibitory profiles of the selected compounds are summarized in Table 1.

\subsection{Cytotoxicity activity}

All compounds were subjected to determine their in-vitro cytotoxicity employing L5178Y and HCT116 cell lines.

In the L5178Y cell lines, (+) dibromophakelline (5) and Z-3-bromohymenialdisine (9) showed cytotoxic activities with inhibition of growth $57.0 \%$ and $60.5 \%$, respectively $(10 \mu \mathrm{g} / \mathrm{mL})$. While, the cytotoxicity of $(-)$ clathramide $C(1), Z-$ spongiacidin D (7), Z-hymenialdisine (8) and 3,4-dibromo-1H-pyrrole-2-carbamide (12), was not as prominent (growth inhibition of $25.3 \%, 36.7 \%, 37.0 \%$ and $38.4 \%$, respectively). But, agelongine (2), (+) manzacidin A (3), (-) 3-bromomanzacidin D (4), E-debromohymenialdisine (6), 2-debromostevensine (10), ( \pm ) ageliferin (11), aldisine (13), 2-bromoaldisine (14) and 4-bromo-1H-pyrrole-2-carbamide (15) showed no significant cytotoxic activity in this cell lines. The results were demonstrated in Figure 2.

The cytotoxic activities were further analysed on HCT116 cell lines. In accordance with the results, which obtained in L5178Y cell lines, Z-3bromohymenialdisine (9) and Z-hymenialdisine (8) exerted relatively high toxicity (significant effects at $25 \mu \mathrm{M}$ after $24 \mathrm{~h}$ ), but the other alkaloids, e.g. $(+)$ dibromophakelline (5) showed no toxic effects. A relatively high toxicity was also caused by ( \pm ) ageliferin (11) and to a lesser extent, by $E$-debromohymenialdisine (7). All other compounds analyzed showed no significant cytotoxic effect up to concentrations of $50 \mu \mathrm{M}$. The results were illustrated in Figure 3 .

\section{Discussion}

Marine sponges are of great pharmacological interest due to the diversity of their secondary metabolites. Although, the molecular mode of action of the most metabolites is still unclear, for a substantial number of compounds the mechanisms 
by which they interfere with the pathogenesis of a wide range of diseases has been reported. Distinct metabolites possess antiviral, antitumor, anti-inflammatory, antioxidative, antibiotic or immunosuppressive activity. Due to these important biological activities, sponges have the potential to provide future drugs against diseases like cancer, malaria and inflammatory diseases [2,29].

This study was performed for the first time on the inhibitory effects of the compounds using 21 important protein kinases in-vitro. Despite the molecular similarity of distinct compounds (only marginal changes in structure), the effects of the compounds on protein kinase inhibition remained to be strongly dependent on the distinct structure. Z-spongiacidin $D(7)$ exhibited the highest capacity to inhibit protein kinases. A potent inhibition of ARK5, CDK2/CycA, CDK4/CycD1, VEGFR-2, SAK and PDGFR-beta was detectable by $Z$-spongiacidin $D(7)$. The structurally related, dibromonated compound Z-3-bromohymenialdisine (9) was also very potent, but with a slight different inhibition pattern of compound Z-spongiacidin $D(7)$. The dibrominated compound Z-3-bromohymenialdisine (9), no inhibition of AKT1, ARK5, B-RAF-VE, CDK2/CycA and IGF1-R was detectable, but in case of AURORA-A and FAK a higher inhibitory effect was seen. The compounds Z-hymenialdisine (8) and E-debromohymenialdisine (6) were structurally similar to the previously mentioned compounds, but less potent. Since, E-debromohymenialdisine (6) is a nonbrominated compound, but also possessed protein kinase inhibitory activity, it has to be concluded that this structural element is no necessity.

Protein kinase inhibition by hymenialdisine was already reported [4], especially kinases of the cell cycle seems to be affected. Also, it interacted with CDC2-Like Kinase 1 [30] and showed CHK1-modulatory effects of hymenialdisine analogues [31]. Moreover, it displayed an inhibition of checkpoint kinase by a hymenialdisinederived analogue [32]. This was congruent with our finding of the inhibition of CDK2/CycA and CDK4/CycD1.

The effect of hymenialdisine/hymenialdisine analogues on 60 recombinant kinases was investigated systematically [33]. They reported eleven new targets like p90RSK, KDR, c-Kit, Fes, MAPK1, PAK2, PDK1, PKC 0 , PKD2, Rsk1 and SGK. They also reported these analogues enhanced and/or dramatically altered selectivity relative to hymenialdisine. This result was also congruent to our findings; $Z$ spongiacidin $D(7)$ and Z-3-bromohymenialdisine (9) showed a higher activity compared with Z-hymenialdisine (8). Our study reported an inhibition of ARK5 by Zhymenialdisine, as well as VEGFR-2 and a weaker effect of this compound to inhibit 
AKT1, CDK2/CycA, CDK4/CycD1, FAK, COT, PLK1, SAK and PDGFR-beta. To the best of our knowledge, no report about e.g. inhibition of AURORA-A and AURORA-B by hymenialdisine/hymenialdisine analogues exists in the literature.

This study was further performed to check the effect of protein kinases on the viability of tumor cells. In L5178Y cell lines, the potent compounds (+) dibromophakelline (5) and Z-3-bromohymenialdisine (9) showed a great inhibition of cell growth. While, Z-spongiacidin D (7), Z-hymenialdisine (8) and 3,4-Dibromo-1 Hpyrrole-2-carbamide (12) also showed a moderate inhibition of cell growth. While, the most of compounds were not or only marginaly toxic. The dibrominated compounds viz., (+) Dibromophakelline (5) and Z-3-bromohymenialdisine (9) showed a comparable high toxicity. While, the dibrominated compound ( \pm ) ageliferin (11) showed very weak toxicity. This indicates that no structural activity relationship between brome atoms in these alkaloids and cytotoxicity.

On the other side, Z-hymenialdisine (8), Z-3-bromohymenialdisine (9) and ( \pm ) ageliferin (11) showed the highest cytotoxic effect on HCT116 cell lines. Consequently, this study demonstrated that the monobrominated compound $Z$ hymenialdisine (8) and the dibrominated compound Z-3-bromohymenialdisine (9) had cytotoxic effects against both of these cell lines. To the best of our knowledge, no reported toxicity of ageliferin existed in the literature. No correlation could be drawn to the kinase inhibition experiments and the assays concerning cytotoxicity.

\section{Conclusion}

Fifteen compounds, mostly monobrominated pyrrole alkaloids were isolated from $S$. carteri had protein kinase inhibitory activity and cytotoxicity. The most potent compounds were Z-spongiacidin D (7), Z-hymenialdisine (8) and Z-3bromohymenialdisine (9). From the results of protein kinase inhibition and cytotoxicity displayed a good correlation, as distinct brominated pyrrole alkaloids effectively inhibited distinct protein kinases followed by a toxic mode of action. These compounds may be interesting for a potential use as pharmacological drugs. In conclusion, distinct pyrrole alkaloids isolated from $S$. carteri effectively inhibited distinct protein kinases in-vitro suggesting a potential use as pharmacological drugs.

Acknowledgments: We wish to thank Prof. Victor Wray (Helmholtz Zentrum für Infektionsforschung, Braunschweig, Germany) for supporting us for the identification 
of the compounds. Our great appreciation to the Egyptian Government for a scholarship of Associate lecturer/ Ashraf Nageeb El-Sayed Hamed.

Conflict of interest statement: The authors report no declarations of interest.

\section{References}

1. Vogel G. The inner lives of sponges. Science 2008;320(5879):1028-30.

2. Hamed ANE, Waetjen W, Edrada-Ebel R, Youssef DTA, Wray V, Kamel MS, Proksch P. A New Bioactive Sesquiterpenoid Quinone from the Mediterranean Sea Marine Sponge Dysidea avara, Nat Prod Commun 2013;8(3):289-92.

3. Blunt JW, Copp BR, Munro MH, Northcote PT, Prinsep MR. Marine natural products. Nat Prod Rep 2005;22:15-61.

4. Nguyen TN, Tepe JJ. Preparation of hymenialdisine, analogues and their evaluation as kinase inhibitors. Curr Med Chem 2009;16(24):3122-43.

5. Zhang N, Zhong R, Yan H, Jiang Y. Structural features underlying selective inhibition of GSK3 $\beta$ by dibromocantharelline: implications for rational drug design. Chem Biol Drug Des 2011;77(3):199-205.

6. Tasdemir D, Mallon R, Greenstein M, Feldberg LR, Kim SC, Collins K, Wojciechowicz D, Mangalindan GC, Concepción GP, Harper MK, Ireland CM. Aldisine alkaloids from the Philippine sponge Stylissa massa are potent inhibitors of mitogen-activated protein kinase kinase-1 (MEK-1). J Med Chem 2002;45(2):529-32.

7. Meijer L, Thunnissen AM, White AW, Garnier M, Nikolic M, Tsai LH, Walter J, Cleverley KE, Salinas PC, Wu YZ, Biernat J, Mandelkow EM, Kim SH, Pettit GR. Inhibition of cyclin-dependent kinases, GSK-3beta and CK1 by hymenialdisine, a marine sponge constituent. Chem Biol 2000;7(1):51-63.

8. Breton JJ, Chabot-Fletcher MC. The natural product hymenialdisine inhibits interleukin-8 production in $U 937$ cells by inhibition of nuclear factor-kappaB. $J$ Pharmacol Exp Ther 1997;282(1):459-66.

9. Yamaguchi M, Miyazaki M, Kodrasov MP, Rotinsulu H, Losung $F$, Mangindaan REP, De Voogd NJ, Yokosawa H, Nicholson B, Tsukamoto S. Spongiacidin C, a pyrrole alkaloid from the marine sponge Stylissa massa, functions as a USP7 inhibitor. Bioorg Med Chem Lett 2013;23(13):3884-86. 
10. Supriyono A, Schwarz B, Wray V, Witte L, Müller WE, van Soest R, Sumaryono W, Proksch P. Bioactive alkaloids from the tropical marine sponge Axinella carteri. Z Naturforsch C 1995;50(9-10):669-74.

11. Tasler S, Müller O, Wieber T, Herz T, Krauss R, Totzke F, Kubbutat MHG, Schächtele C. N-substituted 2'-(aminoaryl)benzothiazoles as kinase inhibitors: Hit identification and scaffold hopping. Bioorg Med Chem Lett 2009;19(5):1349-56.

12. Aly AH, Edrada-Ebel R, Indriani ID, Wray V, Müller WEG, Totzke F, Zirrgiebel U, Schächtele C, Kubbutat MHG, Lin WH, Proksch P, Ebel R. Cytotoxic metabolites from the fungal endophyte Alternaria sp. and their subsequent detection in its host plant Polygonum senegalense. J Nat Prod 2008;71(6):972-80.

13. Edrada R, Proksch P, Wray V, Witte L, Müller WEG, Van Soest RWM. Four new bioactive manzamine-type alkaloids from the Philippine marine sponge, Xestospongia ashmorica. J Nat Prod 1996;59(11):1056-60.

14. Mosmann T. Rapid colorimetric assay for cellular growth and survival: application to proliferation and cytotoxicity assays. J Immunol Methods 1983;65(1-2):55-63.

15. Limper C, Wang Y, Ruhl S, Wang Z, Lou Y, Totzke F, Kubbutat MH, Chovolou $\mathrm{Y}$, Proksch P, Wätjen W. Compounds isolated from Psoralea corylifolia seeds inhibit protein kinase activity and induce apoptotic cell death in mammalian cells. J Pharm Pharmacol 2013;65(9):1393-1408.

16. Cafieri F, Fattorusso E, Taglialatela-Scafati O. Novel bromopyrrole alkaloids from the sponge Agelas dispar. J Nat Prod 1998;61(1):122-25.

17. Cafieri F, Fattorusso E, Mangoni A, Taglialatela-Scafati O. A novel bromopyrrole alkaloid from the sponge Agelas longissima with antiserotonergic activity. Bioorg Med Chem Lett 1995;5(8):799-804.

18. Kobayashi J, Kanda F, Ishibashi M, Shigemori H. Manzacidins A-C, Novel Tetrahydropyrimidine Alkaloids from the Okinawan Marine Sponge Hymeniacidon sp. J Org Chem 1991;56(14):4574-6.

19. Tsukamoto S, Tane K, Ohta T, Matsunaga S, Fusetani N, van Soest RWM. Four new bioactive pyrrole-derived alkaloids from the marine sponge Axinella brevistyla. J Nat Prod 2001;64(12):1576-8.

20. De Nanteuil G, Ahond A, Gulhem J, Poupat C, Tran Huu Dau E, Potier P, Pusset M, Pusset J, Laboute $P$. Invertebres marins du lagon neo-caledonien- 
$V^{1}$ : Isolement et Identification Des Metabolites D'une Nouvelle Espece De Spongiaire, Pseudaxinyssa Cantharella. Tetrahedron 1985;41(24):6019-33.

21. Eder C, Proksch P, Wray V, Steube K, Bringmann G, van Soest RWM, Elisabeth S, Limy F, Pattisina A, Wiryowidagdo S, Moka W. New alkaloids from the indopacific sponge Stylissa carteri. J Nat Prod 1999;62(1):184-7.

22. Inaba K, Sato H, Tsuda M, Kobayashi J. Spongiacidins A-D, new bromopyrrole alkaloids from Hymeniacidon sponge. J Nat Prod 1998;61(5):693-5.

23. Kobayashi J, Tsuda M, Murayama T, Nakamura H, Ohizumi $\mathrm{Y}$, Ishibashi M, Iwamura M, Ohta T, Nozoe S. Ageliferins, potent actomycin ATPase activators from the Okinawan marine sponge Agelas sp. Tetrahedron 1990;46(16):557986.

24. Hassan W, Elkhayat ES, Edrada-Ebel R, Ebel R, Proksch P. New bromopyrrole alkaloids from the marine sponges Axinella damicornis and Stylissa flabelliformis. Nat Prod Commun 2007;2(11):1149-54.

25. Schmitz FJ, Gunasekera SP, Lakshmi V, Tillekeratne LMV. Marine Natural Products: pyrrololactams from several sponges. J Nat Prod 1985;48(1):47-53.

26. Iwagawa $T$, Kaneko $M$, Okamuro $H$, Nakatani $M$, van Soest RWM. New alkaloids from the Papua New Guinean sponge Agelas nakamurai. J Nat Prod 1998;61(10):1310-12.

27. Mancini I, Guella G, Amade P, Roussakis C, Pietra F. Hanishin, a semiracemic, bioactive $\mathrm{C}_{9}$ alkaloid of the Axinellida sponge Acanthella carteri from the Hanish Islands., A Shunt Metabolite? Tetrahedron Lett 1997;38(35):6271-4.

28. Stierle DB, Faulkner DJ. Metabolites of the marine sponge Laxosuberites species. J Org Chem 1980;45(24):4980-2.

29. Sipkema D, Franssen MC, Osinga R, Tramper J, Wijffels RH. Marine sponges as pharmacy. Mar Biotechnol 2005;7(3):142-62.

30. Jain P, Karthikeyan C, Moorthy NS, Waiker DK, Jain AK, Trivedi P. Human CDC2-like kinase 1 (CLK1): a novel target for Alzheimer's disease. Curr Drug Targets 2014;15(5):539-50.

31. Parmentier J, Portevin B, Golsteyn RM, Pierré A, Hickman J, Gloanec P, De Nanteuil G. Synthesis and CHK1 inhibitory potency of hymenialdisine analogues. Bioorg Med Chem Lett 2009;19(3):841-4. 
32. Sharma V, Tepe JJ. Potent inhibition of checkpoint kinase activity by a hymenialdisine-derived indoloazepine. Bioorg Med Chem Lett 2004;14(16):4319-21.

33. Wan Y, Hur W, Cho CY, Liu Y, Adrian FJ, Lozach O, Bach S, Mayer T, Fabbro D, Meijer L, Gray NS. Synthesis and target identification of hymenialdisine analogs. Chem Biol 2004;11(2):247-59. 


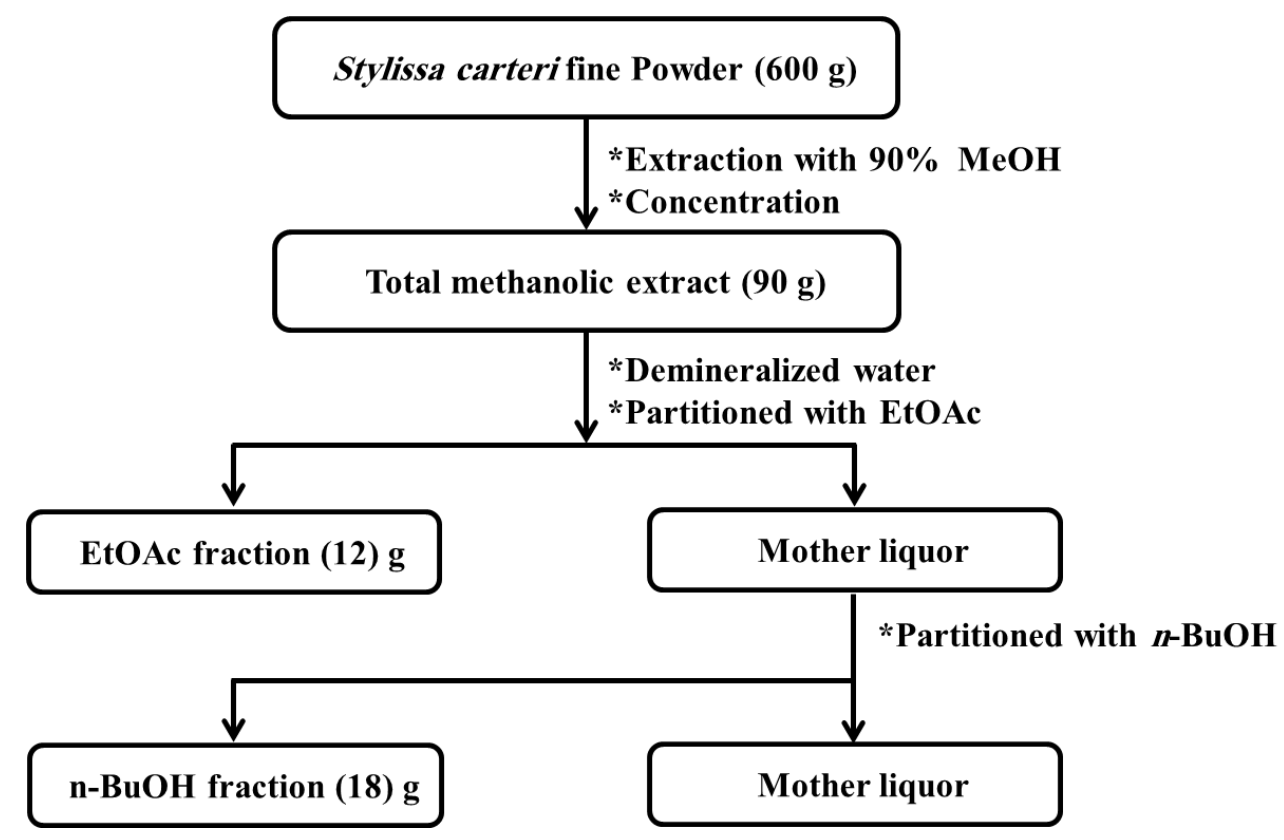

Scheme 1a. Extraction and fractionation of the total methanol extract of $S$. carteri sponge. 


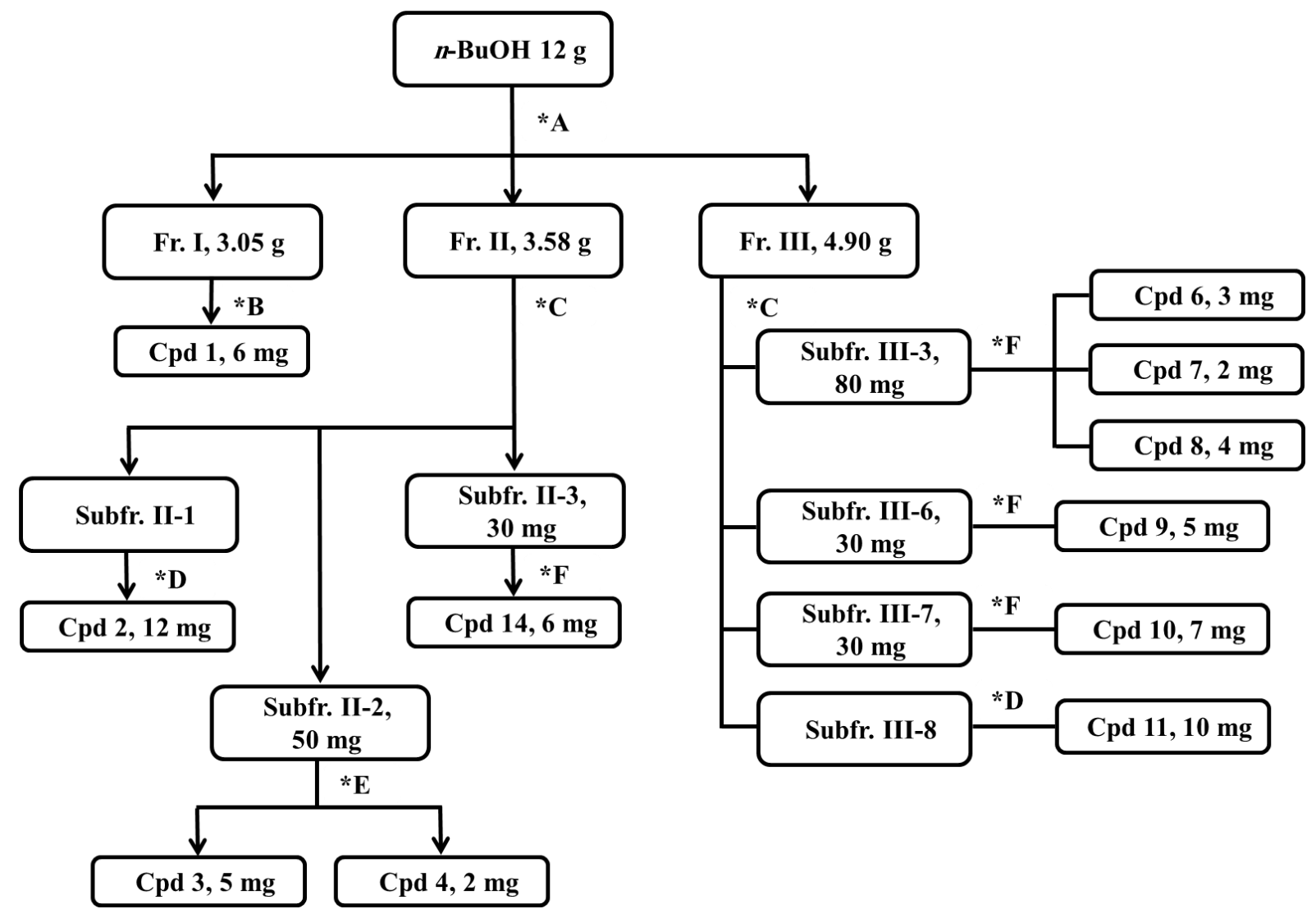

Scheme 1b. Chromatographic fractionation of $n$-butanol fraction of $S$. carteri sponge. 


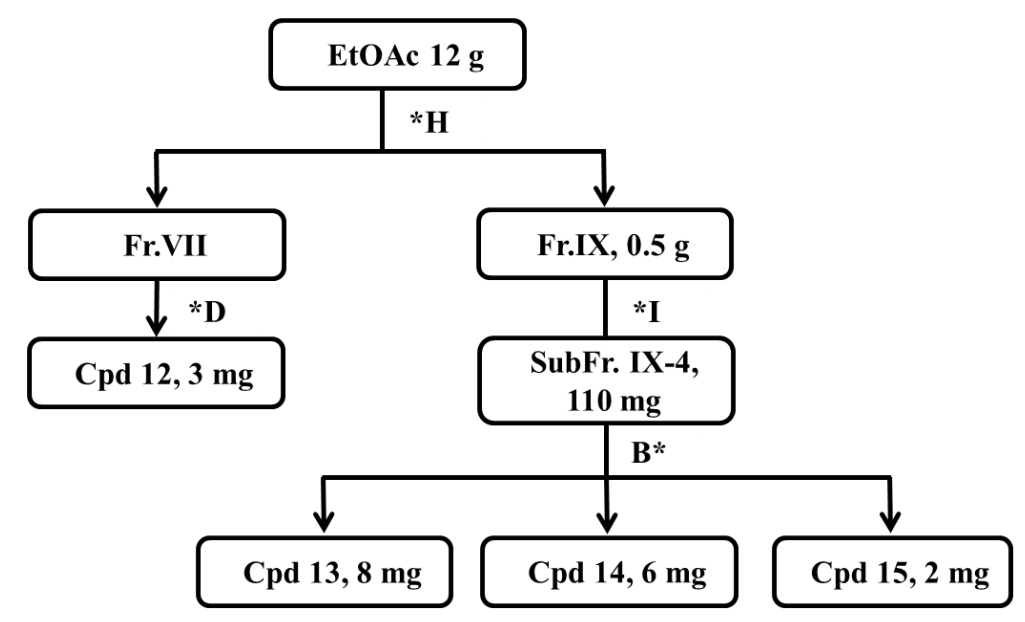

Scheme 1c. Chromatographic fractionation of EtOAc fraction of S. carteri sponge.

The chromatographic techniques abbreviations in the three schemes:

*A: Column chromatography on sephadex LH-20 using MeOH as mobile phase.

*B: Column chromatography on RP-18 using acetonitrile as a mobile phase.

*C: MPLC on RP-18 using $\mathrm{H}_{2} \mathrm{O} / \mathrm{MeOH}$ gradient elution.

*D: Precipitation by time.

*E: Column chromatography on RP-18 using acetonitrile $/ \mathrm{H}_{2} \mathrm{O}(3: 7)$ as a mobile phase.

*F: Semi-preparative HPLC on RP-18 using nanopure $\mathrm{H}_{2} \mathrm{O} / \mathrm{MeOH}$ gradient elution.

*G: MPLC on RP-18 using $\mathrm{H}_{2} \mathrm{O} / \mathrm{MeOH}(7.5: 2.5)$ as a mobile phase.

*H: VLC on silica gel, using gradient elution consisting of different portions of $n$ Hexane $/ \mathrm{CH}_{2} \mathrm{Cl}_{2}$ to $\mathrm{CH}_{2} \mathrm{Cl}_{2} / \mathrm{MeOH}$.

*I: Column chromatography on Silica gel using $\mathrm{CH}_{2} \mathrm{Cl}_{2} / \mathrm{MeOH}$ gradient elution. 

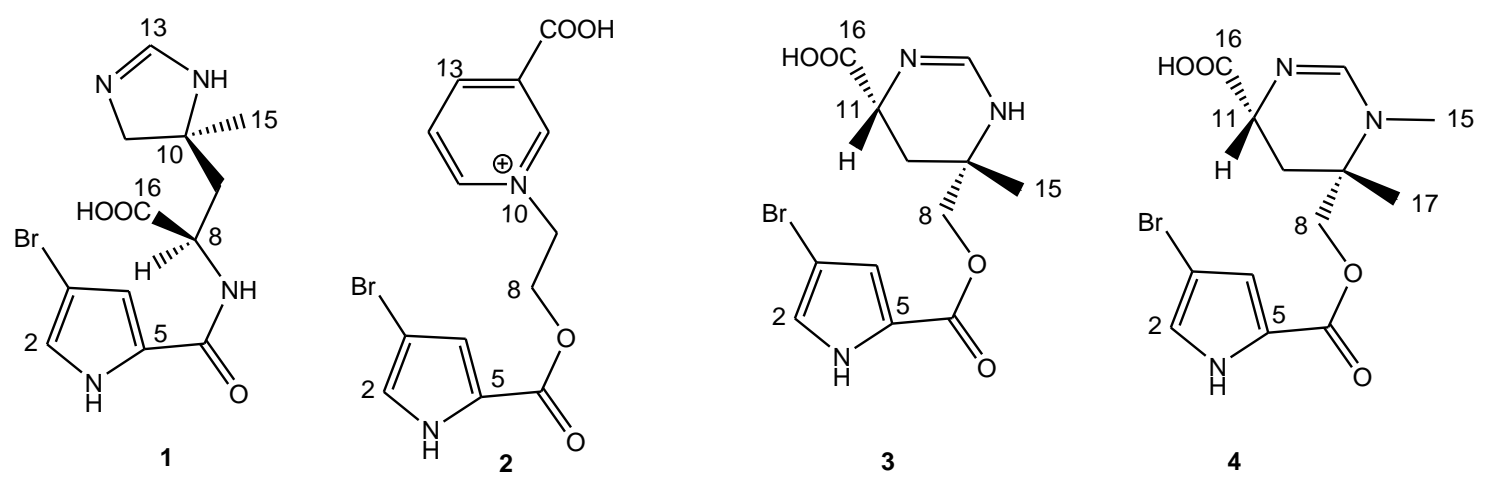

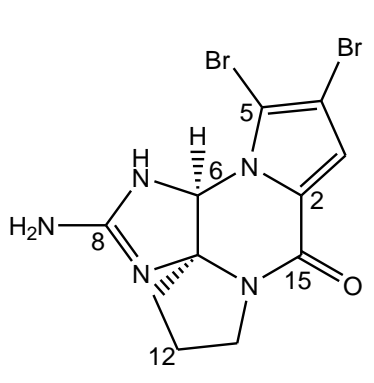

5<smiles>NC1=NC(=O)/C(=C2/CCNC(=O)c3[nH]ccc32)N1</smiles><smiles>O=C1NC(=O)C(=C2CCNC(=O)c3[nH]c(Br)cc32)N1</smiles><smiles>[2H]c1c(Br)[nH]c2c1C(=C1NC(N)=NC1=O)CCNC2=O</smiles><smiles>Nc1nc(C2=CCNC(=O)c3[nH]cc(Br)c32)c[nH]1</smiles>

10<smiles>Nc1ncc(C2c3[nH]c(N)nc3CC(CNC(=O)c3cc(Br)c[nH]3)C2CNC(=O)c2cc(Br)c[nH]2)[nH]1</smiles><smiles>[R]c1c(C(N)=O)[nH]c([TlH])c1Br</smiles>
$12 \mathrm{R}=\mathrm{Br}$
$15 \mathrm{R}=\mathrm{H}$<smiles>Cc1cc2c([nH]1)C(=O)NCCC2=O</smiles>
$13 \mathrm{R}=\mathrm{H}$
$14 \mathrm{R}=\mathrm{Br}$ $4 \mathrm{R}=\mathrm{Br}$

Figure 1: Chemical structures of the isolated compounds from $S$. carteri sponge. 


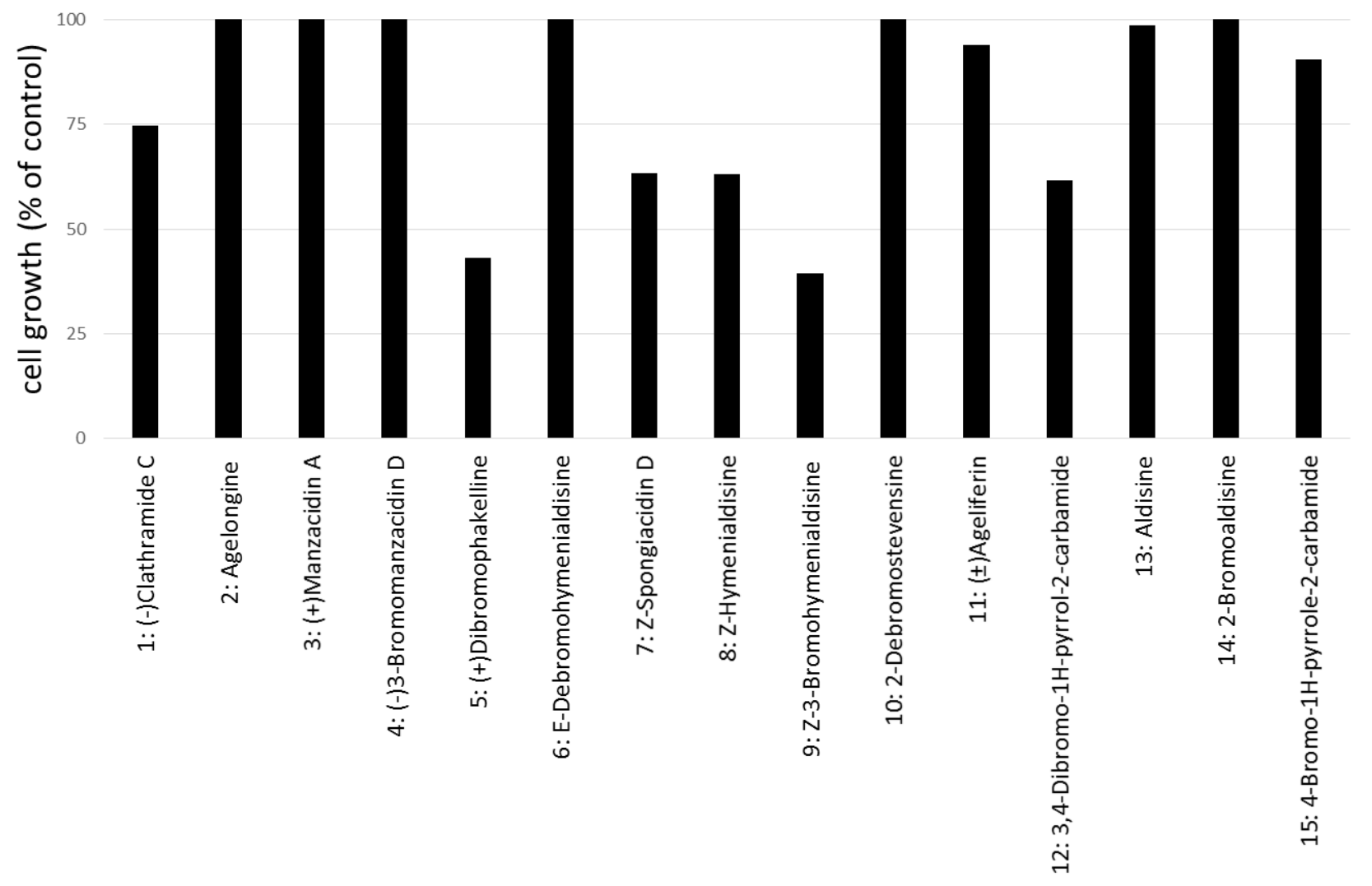

Figure 2: Cytotoxicity of the isolated compounds in L5178Y lymphoma cells from S. carteri sponge. 

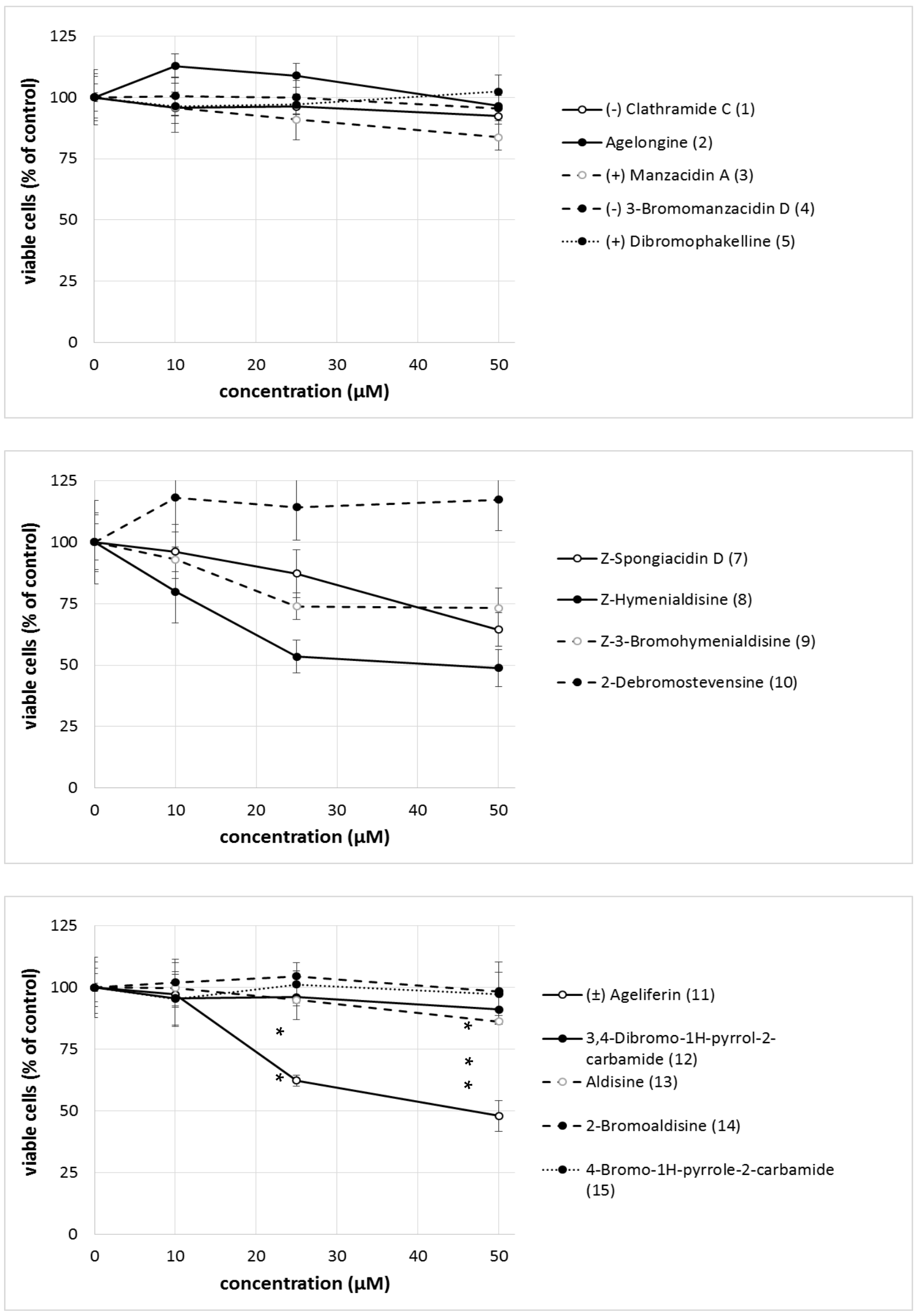

Figure 3: Cytotoxicity of the isolated compounds in HCT116 colon carcinoma cells from $S$. carteri sponge. 
Table 1. Inhibition of protein kinase activity of the isolated compounds from S. carteri sponge.

\begin{tabular}{|c|c|c|c|c|c|c|c|c|c|c|c|c|c|c|c|c|c|c|c|c|c|}
\hline Compound/Protein kinase & 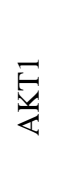 & $\frac{n}{2}$ & 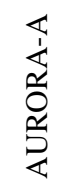 & 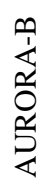 & 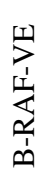 & 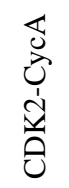 & 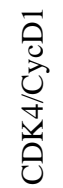 & 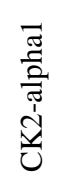 & 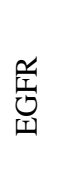 & 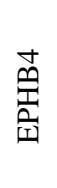 & 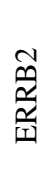 & 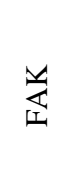 & $\frac{\frac{r}{1}}{\frac{1}{0}}$ & $\frac{\mathscr{z}}{\sim}$ & $\begin{array}{l}\stackrel{̃}{1} \\
\frac{1}{0} \\
\frac{1}{>}\end{array}$ & 8 & $\frac{\vec{a}}{\vec{a}}$ & 宸 & $\begin{array}{l}\frac{\alpha}{1} \\
\text { Ż }\end{array}$ & $\stackrel{5}{\Sigma}$ & $\begin{array}{l}\frac{\pi}{0} \\
\frac{0}{1} \\
\frac{1}{1} \\
0 \\
0 \\
0\end{array}$ \\
\hline (-) Clathramide C (1) & - & - & ++ & + & - & - & - & - & - & - & - & - & - & - & - & - & - & - & - & - & - \\
\hline Agelongine (2) & + & - & - & - & - & - & - & - & - & - & - & - & - & - & - & - & - & - & - & - & - \\
\hline (+) Manzacidin A (3) & + & - & - & - & - & - & - & - & - & - & - & - & - & - & - & - & - & - & - & - & - \\
\hline E-Debromohymenialdisine (6) & - & + & - & - & - & + & + & - & - & - & - & - & - & - & + & - & + & + & - & - & + \\
\hline Z-Spongiacidin $\mathrm{D}(\mathbf{7})$ & + & ++ & + & - & + & ++ & ++ & - & - & - & - & + & + & + & ++ & + & + & ++ & - & - & ++ \\
\hline Z-Hymenialdisine $(\mathbf{8})$ & + & ++ & - & - & - & + & + & - & - & - & - & + & - & - & ++ & + & + & + & - & - & + \\
\hline Z-3-Bromohymenialdisine (9) & - & - & ++ & + & - & - & ++ & - & - & - & - & ++ & - & + & ++ & + & + & ++ & - & - & ++ \\
\hline $\begin{array}{l}\text { 3,4-Dibromo-1H-pyrrole-2- } \\
\text { carbamide } \mathbf{( 1 2 )}\end{array}$ & - & - & - & + & - & - & - & - & + & - & - & + & + & + & + & - & - & - & - & - & - \\
\hline
\end{tabular}

++ : Residual kinase activity $>20 \%$ and $\leq 60 \% .+$ : Residual kinase activity $>60 \%$ and $\leq 80 \%$. $\quad-:$ Resity
Activity on various protein kinases based on $\mathrm{IC}_{50}(\mu \mathrm{g} / \mathrm{mL})$, Compound tested (concentration: $1 \mu \mathrm{g} / \mathrm{mL}$ ). 